\title{
Erythrotrichopeltis, eine neue Gattung der Erythropeltidaceae (Bangiophyceae, Rhodophyta)
}

\author{
P. Kornmann \\ Biologische Anstalt Helgoland (Meeresstation); D-2192 Helgoland, \\ Bundesrepublik Deutschland
}

\begin{abstract}
Erythrotrichopeltis, a new genus of the Erythropeltidaceae (Bangiophyceae, Rhodophyta). In culture experiments Erythropeltis discigera (Berth.) Schmitz and Erythrotrichia discigera Berth. proved to be heteromorphous stages in the life history of the same entity, incorporated into the new genus Erythrotrichopeltis. There is no obligate alternation of generations, the peltoid and the trichoid phase both propagate asexually by monospores. In addition, the trichoid phase releases spermatia in abundance, while carpogonia cannot be distinguished from vegetative cells. Thus far, the present statements are in full accordance with Berthold's observations, made at Naples more than 100 years ago, which have, however, recently been challenged. Moreover, the cultivation experiments made supplement Berthold's results as to the post fertilization process. A few days after the appearance of spermatia, peltoid plants are met with among the filamentous ones: they obviously originate from carpospores. The transition of the peltoid to the trichoid phase, at first observed accidentally in 3-month old moribund cultures, was reconstructed on more than one occasion by designed experiments. This study was started with discoid plants isolated from crude cultures of sublitoral algae from Helgoland (North Sea). Neither Erythrotrichia discigera Berth. nor Erythropeltis discigera (Berth.) Schmitz were ever found in the field around Helgoland. Recognized by Batters to be identical with Bangia ciliaris Carmichael, the name of the typical representative of the new genus is Erythrotrichopeltis ciliaris (Carm. ex Harv. in Hook.) nov. comb.
\end{abstract}

\section{EINLEITUNG}

Der erstmalige Fund scheibenförmiger Thalli von Erythropeltis discigera (Berth.) Schmitz in Rohkulturen mit Algen aus dem Helgoländer Sublitoral war schon bemerkenswert, aber ein fädiger Überzug in ihren drei Monate alten, unbeachtet beiseite gestellten Stammkulturen war völlig überraschend. In frischer Nährlösung wuchsen die Fädchen rasch zu fertilen Pflanzen heran. Sie stimmten mit Erythrotrichia discigera Berthold überein, die ebenfalls bei Helgoland in der Natur noch niemals beobachtet worden ist. Das unbeabsichtigte Kulturexperiment bestätigt einen in Bertholds (1882) Diagnose von Erythrotrichia discigera ausgesprochenen, aber seitdem noch nicht bewiesenen Zusammenhang. Ziel unserer Kulturversuche war es, die genetische Verknüpfung der heteromorphen Erscheinungsformen aufzuklären.

Es ist zweckmäßig, mit einigen Bemerkungen zur Nomenklatur zu beginnen. Berthold (1882) und Hamel (1924) gliedern die Erythrotrichia-Arten nach dem Fehlen oder Vorhandensein einer Basalscheibe in zwei Gruppen. Kylin (1956), einer Anregung von Howe (1914) folgend, geht einen Schritt weiter: der Gattung Erythrotrichia teilt er die Arten zu, deren Fäden einzeln am Substrat haften, während Erythropeltis die Arten 
aufnehmen sollte, deren Fäden einer Basalscheibe entspringen. Der letztere Vorschlag Kylins wurde nicht angenommen, hatte doch Schmitz (1896) seine Gattung Erythropeltis ausdrücklich als einschichtige Scheibe definiert. Sie soll auch weiter auf Formen dieses Typs beschränkt sein. Für Erythrotrichia-Arten mit primärer Basalscheibe wird die neue Gattung Erythrotrichopeltis eingeführt. Ihre einzige bisher entwicklungsgeschichtlich untersuchte Art, Erythrotrichia discigera Berthold, ist heteromorph; nach Batters (1900) ist sie identisch mit Bangia ciliaris Carmichael. Für die typische Art, unser Untersuchungsobjekt, ergibt sich daher der Name Erythrotrichopeltis ciliaris (Carm.) nov. comb.

\section{Erythrotrichopeltis nov. gen.}

Diagnos e: Heteromorphe Gattung der Erythropeltidaceen. Die Fäden des aufrechten Thallus - er wurde als Erythrotrichia discigera Berth. beschrieben - entspringen büschelig einer Basalscheibe; der scheibenförmige Thallus - als Erythropeltis discigera (Berth.) Schmitz beschrieben - ist einschichtig dem Substrat angeheftet. Monosporen werden von der scheibenförmigen und von der aufrechten Pflanze in der für die Erythropeltidaceen kennzeichnenden Weise gebildet. In gleicher Art werden in den Zellen des aufrechten Thallus kleine, deutlich gefärbte Spermatien erzeugt. Karpogone entstehen unmittelbar aus vegetativen Zellen. Aus den Karposporen geht die scheibenförmige Generation hervor.

Herkunft: Erythropeltis-Scheiben traten selten in Rohkulturen von Algen aus dem Helgoländer Sublitoral auf. Erythrotrichia-Fäden entstanden in absterbenden Erythropeltis-Kulturen.

Typ ische Art: Erythrotrichopeltis ciliaris (Carm. ex. Harv. in Hook.) nov. comb., als synonym angesehen mit Erythrotrichia discigera Berth. und Erythropeltis discigera (Berth.) Schmitz.

D i a g o s is: Genus heteromorphus Erythropeltidacearum. Filamenta thalli erecti - nomine Erythrotrichia discigera Berth. descripta est - a disco basale gregatim surgentes; thallus discoideus - nomine Erythropeltis discigera (Berth.) Schmitz descripta est monostromaticus, substrato adfixus. Monosporae tum in statu discoidea tum in fronde erecta modo Erythropeltidacearum evolutae. In cellulis status trichoidei eodem modo spermatiae minutae, dilute coloratae, gignentes. Carpogoniae e transformatione immediata cellularum vegetativarum efformatae. Frons discoidea ex carposporis nata.

Origo: Disci Erythropeltidis rare occurrunt in culturis crudis algarum sublitoralium insulae Helgolandiae. Filamenta Erythrotrichii in culturis in statu moriendi Erythropeltidis orta sunt.

Species typica: Erythrotrichopeltis ciliaris (Carm. ex Harv. in Hook.) nov. comb., synonyme aestimata Erythrotrichia discigera Berth. et Erythropeltis discigera (Berth.) Schmitz.

\section{MATERIAL UND METHODE}

Ausgangsmaterial waren im Dezember 1982 sowie im Januar 1983 und 1984 angelegte Rohkulturen mit Chaetomorpha melagonium und Rhodomela aus dem Sublitoral; aus ihnen wurden die in nur geringer Anzahl gefundenen Scheiben von Erythropeltis discigera (Berth.) Schmitz isoliert. Im allgemeinen wurden die Scheiben und die aus ihnen entstandenen fädigen Thalli auf dem Boden von Plastikschalen kultiviert, 
gelegentlich auch auf lebenden Substraten, Bryopsis hypnoides und Chaetomorpha linum, angesiedelt. Als Kulturmedium diente im allgemeinen Nährlösung nach Provasoli mit einem Zusatz von Erdabkochung. Die Temperatur schwankte um $15^{\circ} \mathrm{C}$, es wurden täglich 14 Stunden Licht in einer Stärke von 1000-2000 Lux je nach Abstand von der Leuchtstofflampe geboten.

\section{KULTURVERSUCHE}

\section{Der scheibenförmige Thallus}

Die Ontogenese der Scheibe ist in einer 7 tägigen Serie von Aufnahmen dargestellt (Abb. 1). Die Keimlinge aus den ziemlich einheitlich $9 \mu \mathrm{m}$ dicken Sporen sind schon

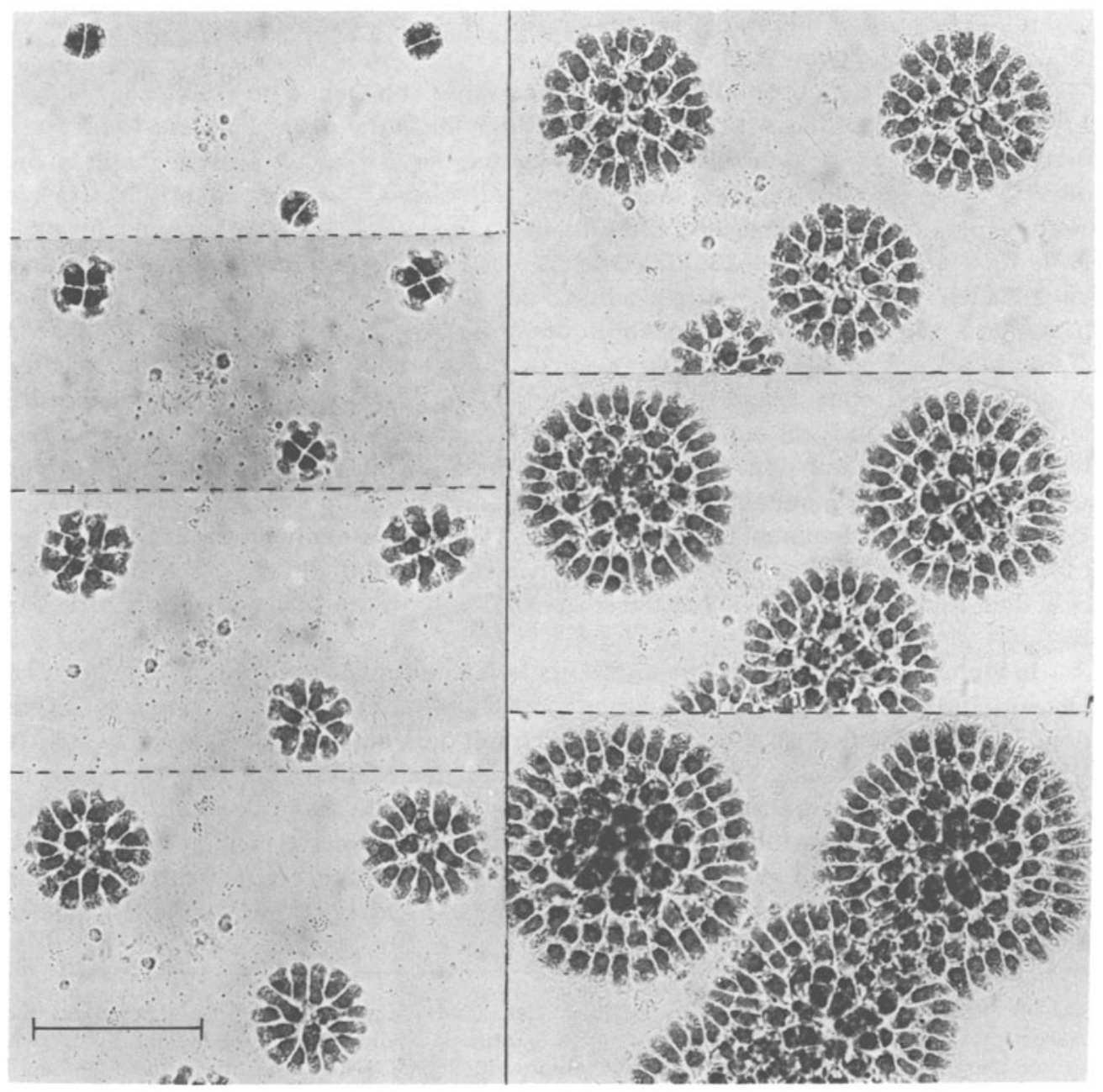

Abb. 1. Erythrotrichopeltis ciliaris. Entwicklung des scheibenförmigen Thallus vom 2 , bis zum 8 . Tage. Maßstrecke $=50 \mu \mathrm{m}$ 
nach einem Tag zweizellig. Das zwei Tage alte Scheibchen besteht aus vier kreuzweise geordneten Zellen, die peripher dichotom ausgebuchtet sind. Sie teilen sich durch eine schräg verlaufende Wand, so daß eine kürzere Zelle seitlich einer längeren aufsitzt. In den sich durch Randwachstum vergrößernden Scheiben entstehen polygonale Zellen in der Scheibenmitte durch konzentrisch angelegte Wände.

Nach 8 bis 9 Tagen werden die Scheiben fertil. Aus den Monosporen entsteht eine den Eltern gleiche Generation. Regelmäßig treten auf dem Boden von Plastikschalen auch höckerige Formen auf, in älteren Kulturen werden sie häufiger. Sie können als dichte Zellhaufen den Scheiben aufsitzen (Abb. 2 D) oder auch selbständige unregelmäBige Zellaggregate bilden (Abb. $2 \mathrm{~A}$ ). Weniger häufig entstehen auch fädige Keimlinge, deren Zellen sich aber bald zu Knäueln verdicken, sie verlängern sich niemals zu Erythrotrichia-Fäden (Abb. 2 B, C). Dünnfädige Algen oder Baumwollfäden werden mantelförmig umhüllt, eine bei scheibenförmigen Algen ganz allgemein zu beobachtende Erscheinung (Abb. $2 \mathrm{~F}$ ).

Unsere Alge gleicht in allen Einzelheiten einer von Dangeard (1968) zur Gattung Erythrocladia gestellten Art, die er wegen ihrer höckerigen Erscheinungsformen mit dem Epithet "gibber" kennzeichnete. Von den beiden unterschiedenen Formen ist nur die "forme à grandes cellules marginales" mit unserer Scheibe identisch, wie ein Vergleich mit seinen zahlreichen Abbildungen ohne jeden Zweifel erkennen läßt (1968, Pl. I, fig. 9-15; Pl. II, fig. 14-23; Pl. VI, A, B; Pl. IX, C). Den Knäueln können manchmal auch Fäden entspringen: "parfois même, des filaments partent de ces glomérules" (Dangeard, 1969, p. 6), wie es die Abbildung 2 E darstellt.

Dem kritischen Betrachter müssen solche Kulturen "unnatürlich" vorkommen, und wahrscheinlich treten die knäueligen Thalli bei den als Epiphyten in bewegtem Wasser lebenden Scheiben nicht auf. Einer dieser Faktoren war in Kultur leicht zu bieten: das lebende Substrat. Auf den Stämmchen von Bryopsis hypnoides oder auf Fäden von Chaetomorpha linum gedeihen die scheibenförmigen Thalli vorzüglich, und knäuelige Zellaggregate wurden nicht beobachtet (Abb. 2 G-I). Viele der in großer Zahl erzeugten Monosporen fallen zu Boden, doch setzen sich in unmittelbarer Nähe der fertilen Scheiben sicherlich mehr Sporen fest als es im bewegten Wasser des natürlichen Standorts der Fall wäre.

In vieler Hinsicht sind unsere und Dangeards Kulturen denen ähnlich, die Nichols \& Lissant (1967) bei "Erythrocladia subintegra" erhielten. Ihr Untersuchungsobjekt aus dem Golf von Mexico ist aber nicht identisch mit dem europäischen Typus dieser Art. Vielmehr entspricht die Größe der Zellen und der Monosporen den Dimensionen unserer Scheibengeneration. Die ausgeprägte amöboide Beweglichkeit der Sporen unterscheidet sie wesentlich von unserem Objekt, dessen Sporen wohl geringe Gleitbewegungen machen und sich zum Beispiel in einer Linie längs eines Kratzers auf dem Schalenboden ansammeln können, sie sind aber nicht erkennbar amöboid. Die in

Abb. 2. Erythrotrichopeltis ciliaris. Variabilität des scheibenförmigen Thallus. A Scheiben und knäuelige Zellhaufen in einer 11 Tage alten Kultur. B Fädig-knäueliges Zellaggregat, zwei Aufnahmen in verschiedenem Niveau sind aneinandergesetzt. C Fädiger Keimling und Scheiben, 7 Tage alt. D Zellklumpen auf einer Scheibe. E Fäden aus einem Zellknäuel entspringend. $F$ Mantelförmig umhüllte Baumwollfäden. G, H Scheiben epiphytisch auf Chaetomorpha linum, I auf Bryopsis hypnoides. MaBstrecken: $A, H, I=100 \mu \mathrm{m} ; \mathrm{B}-\mathrm{F}=50 \mu \mathrm{m} ; \mathrm{G}=200 \mu \mathrm{m}$ 


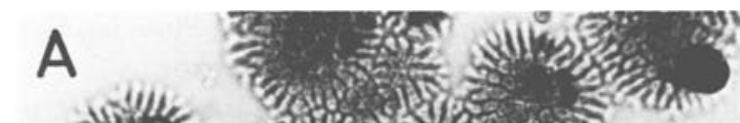

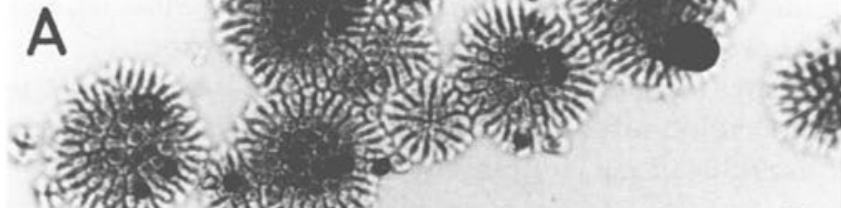

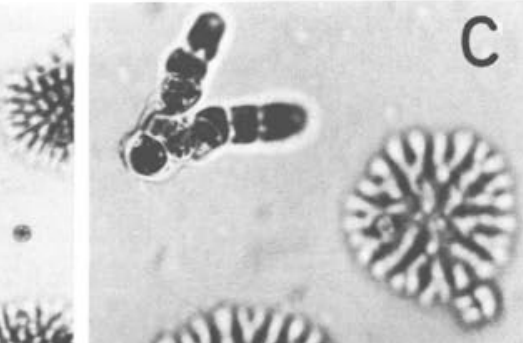

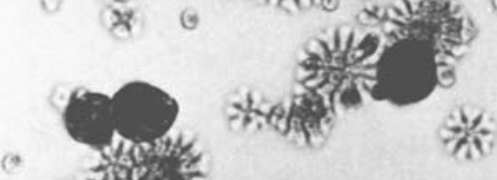

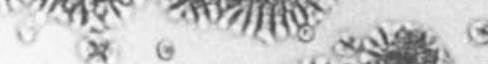

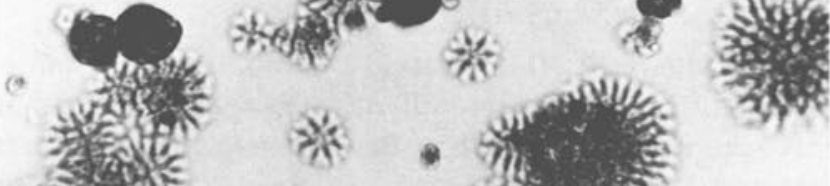
Wibasite andinsus
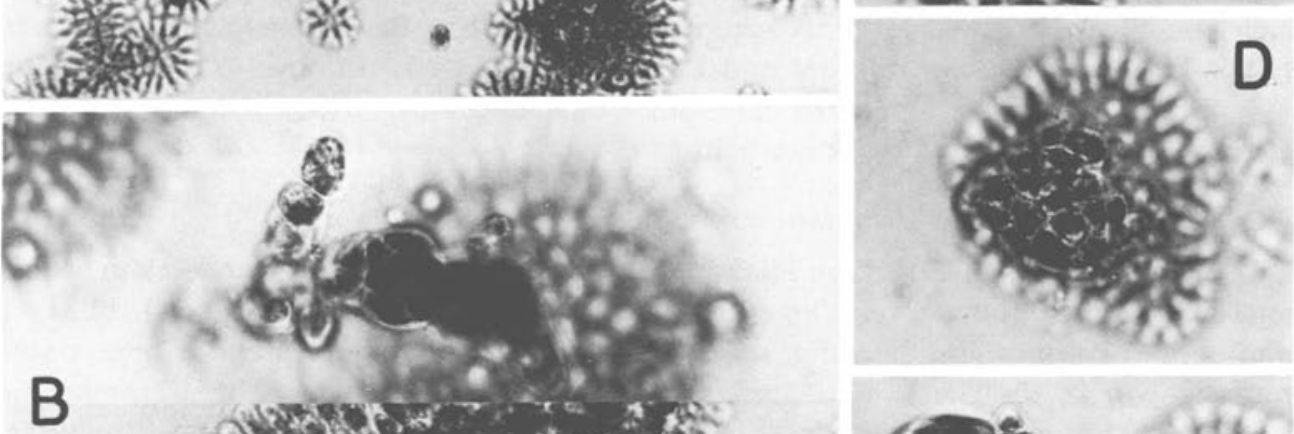

B
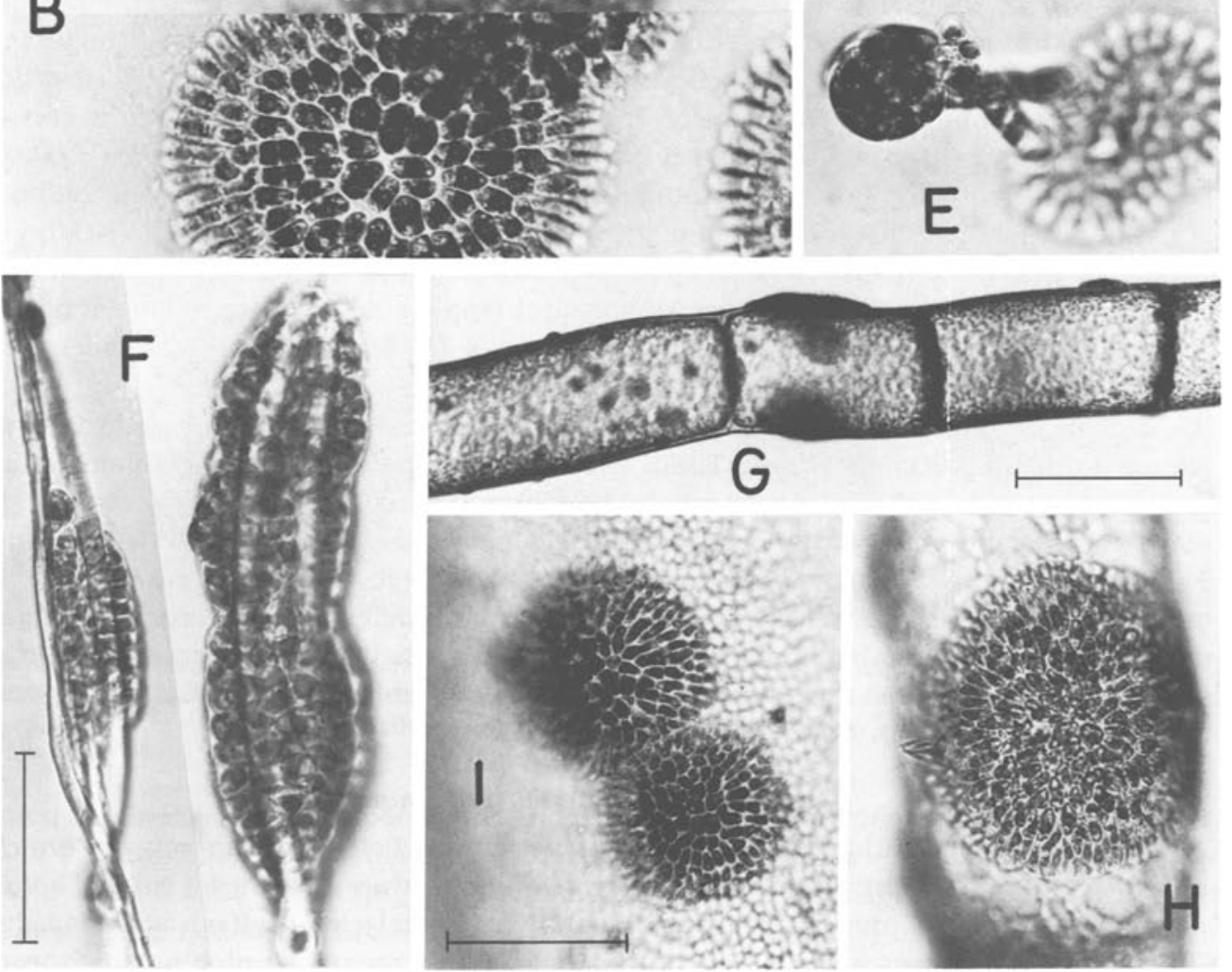
älteren Kulturen auftretenden knäueligen Zellaggregate (Plate II, Fig. 60, Plate VI, Fig. 99-100) mögen wohl denen in Dangeards und unseren Kulturen entsprechen.

Wir schließen die Möglichkeit nicht aus, daß die von Nichols \& Lissant als "Erythrocladia subintegra" bezeichnete Alge keine selbständige Form ist, sondern das scheibenförmige Stadium im Entwicklungszyklus eines fädigen Thallus darstellen könnte.

\section{Der aufrechte Thallus}

\section{Allgemeiner Überblick}

Epiphytisch auf Chaetomorpha kultivierte Pflanzen sind in Abbildung 3A dargestellt. Die Fäden erzeugen etwa vom 10. bis 11. Tag an laufend Monosporen, aus denen sich wieder aufrechte Thalli entwickeln. Wenige Tage später treten die von Berthold (1882) bei Erythrotrichia obscura und E. discigera beobachteten hyalinen Spermatien auf, und schließlich werden auBer den Monosporen auch Sporen gebildet, aus denen die scheibenförmige Generation hervorgeht.

\section{Die ungeschlechtliche Vermehrung}

Die vielen von einer fertilen Pflanze entleerten kugeligen, 10-11 $\mu \mathrm{m}$ dicken Monosporen sinken in einer zugleich ausgeschiedenen Gallerte langsam zu Boden (Abb. 4 A). Auf dem Substrat platten sie sich schon nach wenigen Stunden ab und werden dabei auffallend hell, ihr Umriß wird unregelmäßig (Abb. $5 \mathrm{~A}$ ). Nach einem Tag sind die meisten Keimlinge zweizellig und deutlich von einer hyalinen Haftmembran umgeben (Abb. 4 B). Zwei Tage alte Scheibchen können schon vierzellig sein, ihr Umriß wird durch die periphere Ausbuchtung der Zellen sternförmig (Abb. 4 C). Nach 3 und 4 Tagen entsprossen den Scheiben zwei bis vier aufrechte Fädchen $(D, E)$, die nach 5 Tagen schon mehrzellig geworden sind $(F)$. Zugleich haben sich auch die Basalscheiben durch das Wachstum ihrer Randzellen vergrößert. Sie sind in den einzelnen Kulturen mehr oder weniger tief ausgebuchtet, ganz offenbar liegt hier ein nicht kontrollierbarer morphogenetischer Einfluß des Substrats vor. Breitlappig sind die Randzellen der bis zu 5 Tage alten Jugendstadien unserer Abbildungen $4 \mathrm{D}-\mathrm{G}$, sie können aber auch tief gabelig geteilt sein ( $\mathrm{H}-\mathrm{K})$.

In den einreihig angelegten Erythrotrichia-Fäden treten schon frühzeitig Längswände auf (Abb. $4 \mathrm{~K}$ ). Die älteren Thalli haben im allgemeinen 4 bis 8 nebeneinanderliegende Zellen, so daß der Thallus schmal bandförmig wird (Abb. 3 B-D). Oft entspringt dicht über der Basis ein Seitenzweig, nur ausnahmsweise verzweigt sich der flächige Thallus. Die Thalli sind selten glattrandig, meist etwas eingeschnürt oder zuweilen auch gekerbt, häufig leicht spiralig gedreht. Die primär gebildeten Fäden werden im allgemeinen 2-3 mm, selten bis $6 \mathrm{~mm}$ lang. Aus den Basalscheiben entsprossen zahlreiche sekundäre Fäden, so daß die einzelne Pflanze dicht büschelig wird. Die Entleerung von Monosporen beginnt im Alter von 10 bis 12 Tagen.

\section{Die geschlechtliche Fortpflanzung}

Berthold (1882) berichtete über die geschlechtliche Fortpflanzung von Erythrotrichia obscura. Geschlechtspflanzen, besonders weibliche, waren bei dieser Art und bei $E$. discigera selten und immer mit ungeschlechtlichen gemischt. Vielfach schienen die Fäden rein männlich zu sein, in seltenen Fällen wurden Spermatangien neben Procar- 

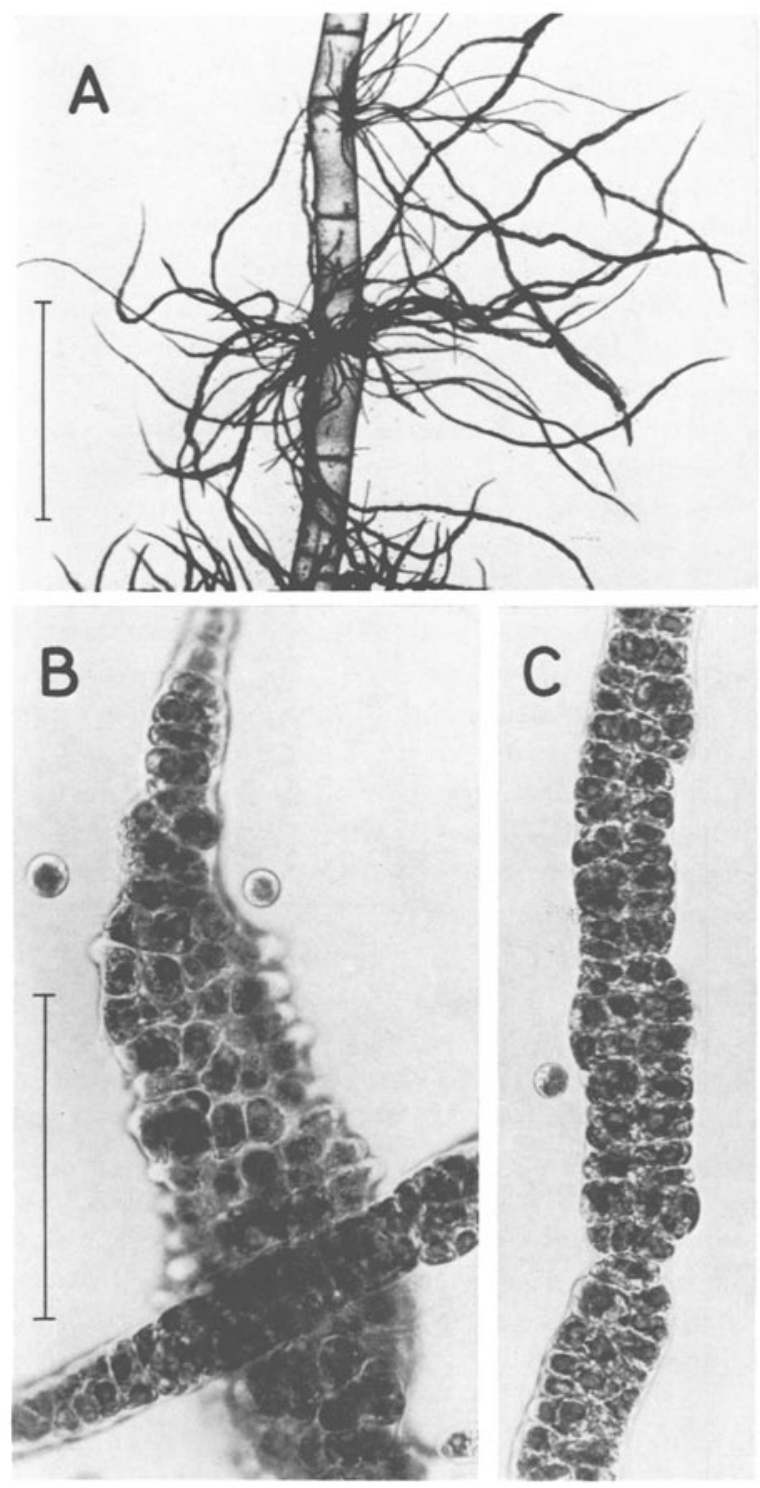

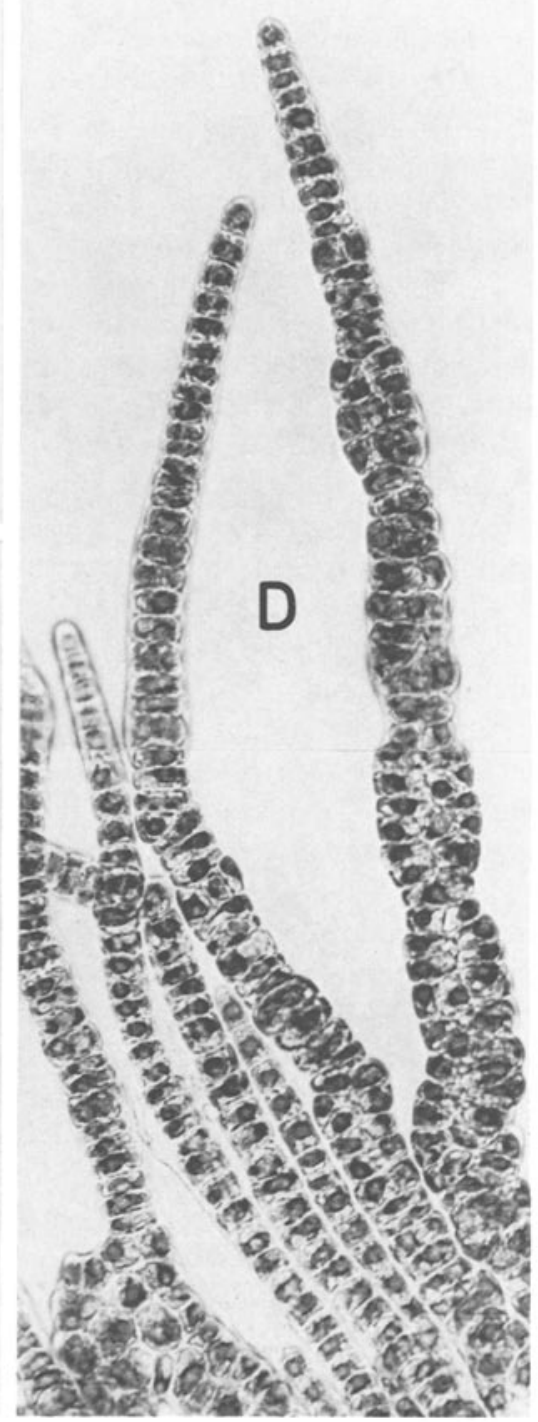

Abb. 3. Erythrotrichopeltis ciliaris. Fädiger Thallus. A Epiphytisch auf Chaetomorpha linum. B-C Fertile Thallusabschnitte und Monosporen. D Verschieden alte Thallusenden eines Büschels. Maßstrecken: $\mathrm{A}=1 \mathrm{~mm}$; $\mathrm{B}-\mathrm{D}=100 \mu \mathrm{m}$

pien gebildet, sonst aber Spermatien und neutrale Sporen auf demselben Faden erzeugt. Die Procarpzellen unterscheiden sich zuerst nicht von den vegetativen Zellen, später wird eine leichte Vorstülpung nach außen gebildet. Die kugeligen Spermatien mit einem gut sichtbaren Chromatophor setzen sich auf der Thallusoberfläche an; durch einen feinen Keimschlauch tritt ihr Inhalt in die weibliche Zelle über. Nach der 


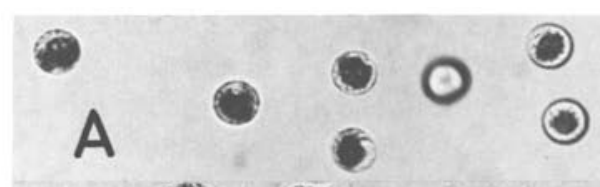

-.

- B

- B

* is

c
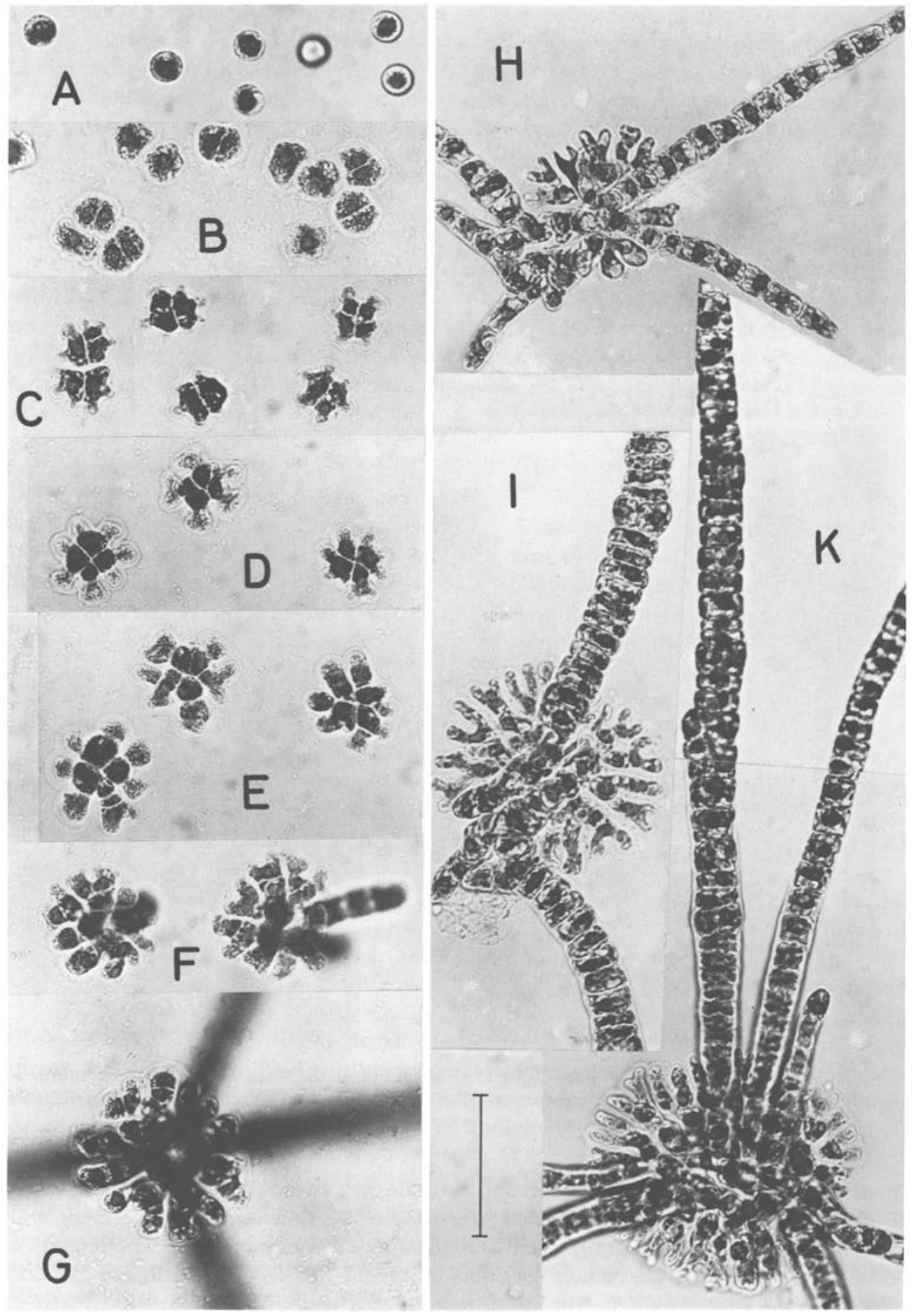
Befruchtung wird eine einzige Cystocarpspore gebildet; ihren Austritt und ihr weiteres Verhalten konnte Berthold nicht beobachten.

Diese Darstellung Bertholds und die entsprechenden Abbildungen sind in die älteren Lehrbücher übernommen worden (Oltmanns, 1922, Fig. 461, 2-5; Fritsch, 1945 , Fig. 144, G-I; Kylin, 1956, Fig. 22, F-J. In Oltmanns' und Kylins Legenden sind die Monosporangien unzutreffend als Spermatangien bezeichnet!). Die neuere Literatur (Dixon, 1973; Dixon \& Murray, 1981) begegnet Bertholds Darstellung mit Zweifel. Offenbar sind seine Beobachtungen an lebendem Material aus dem Formenkreis von Erythrotrichia ciliaris bisher nicht wiederholt worden.

Unsere Untersuchungen bestätigen Bertholds Angaben über die geschlechtliche Vermehrung in vollem Umfang. Wenn auch die Befruchtung nicht direkt beobachtet oder zytologisch nachgewiesen ist, so wird diese Lücke durch das Ergebnis der Kulturversuche überzeugend geschlossen. In mehreren Versuchsreihen wurden Einzelpflanzen bis zu 3 Wochen lang täglich an markierte Stellen der Kulturschalen verlagert. Monosporen wurden vom 11. Tage an während der ganzen Versuchsdauer erzeugt. Die Spermatienbildung beginnt 3-4 Tage später und dauert nur wenige Tage an. Wiederum nach 3 Tagen werden Sporen entleert, aus denen sich Scheiben entwickeln: sie sind 4-5 Tage später leicht zwischen den Keimlingen der fädigen Pflanzen zu erkennen.

Eine der geprüften Einzelpflanzen gehörte zu einem Stamm, der keine Spermatien, wohl aber etwas kleinere Sporen mit dichtem Inhalt entleerte. In der Nachkommenschaft solcher Pflanzen traten keine Scheiben auf; die kleinen Sporen entwickelten sich langsamer zu fädigen Pflanzen als die größeren. Auch dieses Versuchsergebnis läßt darauf schließen, daß die scheibenförmige Generation nur nach einer vorausgegangenen Befruchtung entsteht.

Unsere mikroskopischen Befunde stimmen ebenfalls mit Bertholds Beobachtungen überein. Abbildung $5 \mathrm{~A}$ zeigt Spermatien und Monosporen 18 Stunden nach ihrer Entleerung auf dem Boden einer Kulturschale. Die Monosporen haben sich schon abgeplattet, die kugeligen umhäuteten Spermatien mit deutlichem Chromatophor liegen lose auf dem Boden oder schweben noch. Ihr Durchmesser schwankt zwischen 5 und $9 \mu \mathrm{m}$. Während die kleineren im Laufe weniger Tage zugrunde gehen, bleiben einzelne der größeren Spermatien am Leben. Ihr Chromatophor wächst und füllt die dicker werdenden Zellen weitgehend aus, sie erheben sich, auf einem äußerst zarten Stielchen verankert, in die Flüssigkeit. Es war nicht möglich, ihre Entwicklung länger als 12 Tage zu beobachten, weil sie dann von den herangewachsenen fädigen Thalli und deren Nachkommenschaft überwuchert wurden.

Abbildung 5 B zeigt die in großer Zahl an der Oberfläche eines Fadens angehefteten Spermatien, ganz analog der von Berthold nach dem Leben gezeichneten Figur 24. In den mit Glyzerin versetzten Präparaten sieht man die Keimschläuche der Spermatien in Verbindung mit den als Karpogonien anzusehenden Zellen (Abb. 5 C). Die Vorstülpungen mehrerer Zellen in Abbildung $5 \mathrm{~F}$ (Pfeile) könnten vielleicht als Trichogynen gedeutet werden. Fadenabschnitte mit Spermatangien und einzelnen Monosporangien (Pfeile) sind bei D und E dargestellt, ein Faden mit zahlreichen Monosporangien bei F.

Abb. 4. Erythrotrichopeltis ciliaris. Entwicklung des aufrechten Thallus. A Monosporen. B-F Ein bis fünf Tage alte Keimlinge. G Basalscheibe mit vier aufrechten Fäden. H, I Etwa 7-9 Tage alte Thalli in Aufsicht, $\mathrm{K}$ von unten gesehen. Maßstrecke: $\mathrm{A}-\mathrm{K}=50 \mu \mathrm{m}$ 


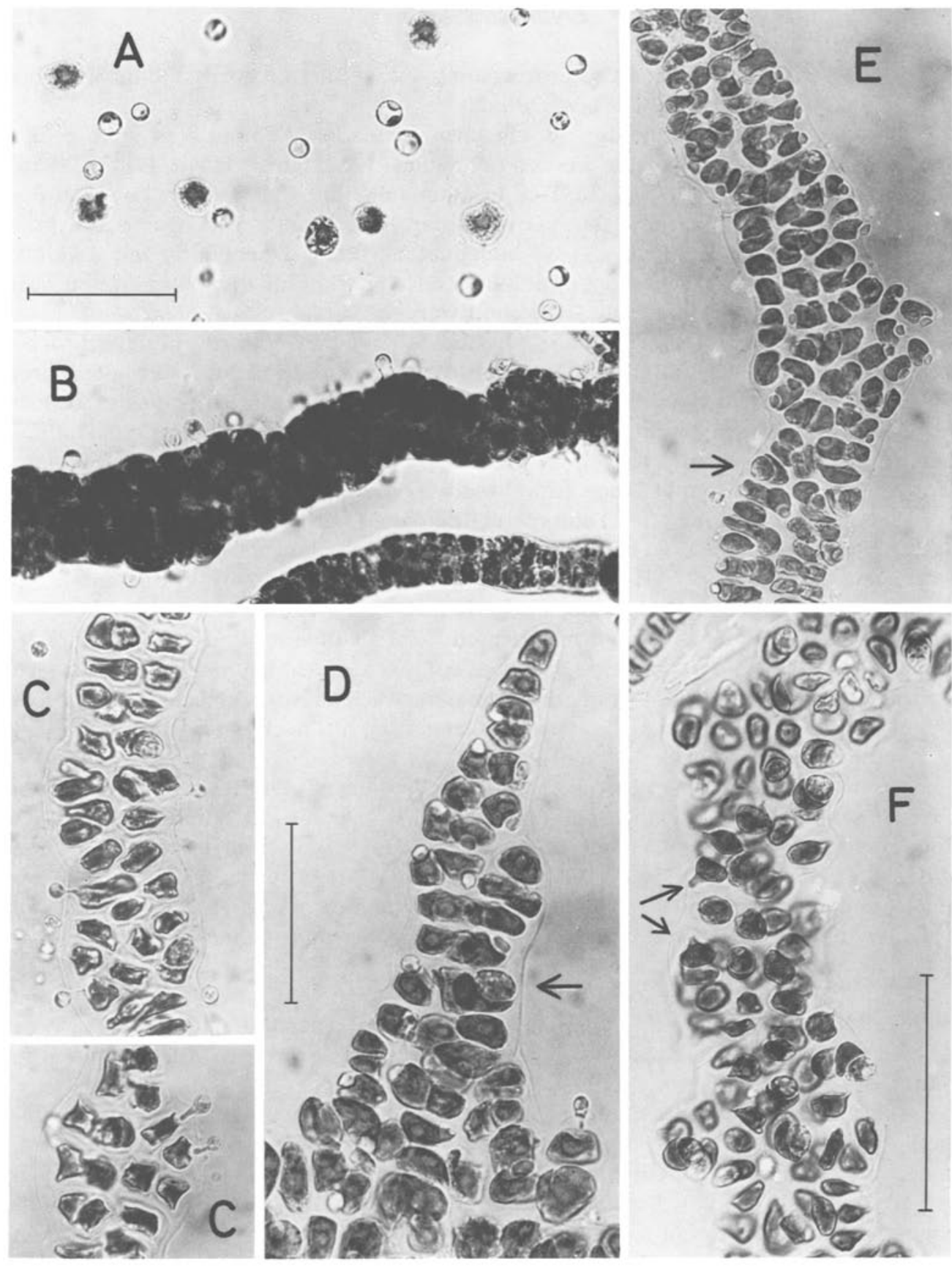

Abb. 5. Erythrotrichopeltis ciliaris. Fruktifikation. A Keimende Monosporen und kugelige, zum Teil noch schwebende Spermatien, 18 Stunden nach der Entleerung. B Auf einem Faden ansitzende und keimende Spermatien, lebend. C-F Nach Fixierung und Zugabe von Glyzerin. C Spermatien mit Befruchtungsschlauch. D, E Thallusstücke mit Spermatangien und einzelnen Monosporangien (Pfeile). F Thallus mit Monosporangien und Prokarpien? (Pfeile). Maßstrecken: $A=50 \mu \mathrm{m} ; \mathrm{B}, \mathrm{E}, \mathrm{F}$ $=100 \mu \mathrm{m} ; \mathrm{C}, \mathrm{D}=50 \mu \mathrm{m}$ 
Bei dieser reichlichen Fruktifikation ist es nicht verwunderlich, daß von den primären Fäden einer Einzelpflanze täglich 100-200 Sporen und Spermatien entleert werden, die sekundär entstehenden Fäden setzen die üppige Fruktifikation fort.

\section{Die Verknüpfung der heteromorphen Generationen}

Die Aufeinanderfolge der heteromorphen Generationen ist nicht obligat, beide können sich selbständig durch Monosporen fortpflanzen (Abb. 7). Während die Herkunft der scheibenförmigen Thalli aus Karposporen wahrscheinlich gemacht werden konnte, bleibt die Entstehung der fädigen aus der scheibenförmigen Generation völlig ungeklärt. Zum ersten Male wurden die Fäden zufällig in überalterten Kulturen gefunden. Die versuchsmäßige Wiederholung führte zu dem gleichen Ergebnis, ließ aber nicht die inneren Zusammenhänge erkennen. Es seien daher nur die näheren Umstände dieser Versuche ausführlich mitgeteilt. In den mit wenigen Monosporen oder einer fertilen Einzelscheibe angelegten Kulturen wurde die Nährlösung nicht erneuert. Nach etwa 4 Wochen war der Boden der $5 \mathrm{~cm}$ großen Plastikschalen zum großen Teil mit einer gallertigen Schicht von miteinander verschmolzenen und übereinandergelagerten Scheiben bedeckt. Die Kulturen wurden zunehmend blasser, und die Sporenbildung hörte auf. Im Zentrum der Kultur löste sich die gallertige Haut vom Substrat und wölbte sich blumenkohlartig auf. Teile der recht festen Gallerte wurden nach 11 Wochen in frische Nährlösung übertragen. Diese Hautstücke waren fast farblos, enthielten aber immer noch Inseln gefärbter Zellkomplexe. Schon nach wenigen Tagen wurden wieder Monosporen gebildet, die sich zu Scheiben entwickelten. Aus einzelnen der dunkler und größer gewordenen Zellaggregate waren nach 16 Tagen Erythrotrichia-Fädchen entsproßt (Abb. 6). Sie wuchsen in frischer Nährlösung rasch heran und wurden fertil. Es wurde nicht versucht, durch Variation der Kulturbedingungen den Übergang von der peltoiden zur trichoiden Phase zu beeinflussen.

Die Lebensgeschichte von Erythrotrichopeltis ciliaris ist in einer schematischen Darstellung veranschaulicht (Abb. 7). Das Ergebnis der Kulturversuche macht auch ohne zytologischen Nachweis einen an die heteromorphen Erscheinungsformen gebundenen Kernphasenwechsel wahrscheinlich. Das Entwicklungsschema von Erythrotrichopeltis entspricht völlig dem einiger Bangia- und Porphyra-Arten (Cole \& Conway, 1980); in dieser Hinsicht unterscheiden sich die Erythropeltidaceen nicht von den Bangiaceen.

\section{TAXONOMISCHER TEIL}

Nachdem die Heteromorphie von Erythrotrichopeltis an dem Beispiel ihrer typischen Art erkannt ist, lassen sich auch andere Erythrotrichia-Arten mit primärer Basalscheibe der Gattung zuführen. Texte und Abbildungen mehrerer Autoren bestätigen ganz offen den im Kulturexperiment aufgedeckten Zusammenhang oder lassen sich leicht in diesem Sinne interpretieren. Dies gilt für Erythrotrichia ciliaris und die synonym beschriebenen Arten ebenso wie für $E$. boryana. Dagegen wird $E$. polymorpha, deren taxonomischer Status zu wenig geklärt ist, nicht in die neue Gattung aufgenommen.

Die Einführung der Gattung Erythrotrichopeltis beseitigt zugleich eine nomenklatorische Verwirrung. Papenfuss (1951, p. 172) wies darauf hin, daß das Binom Erythrotri- 


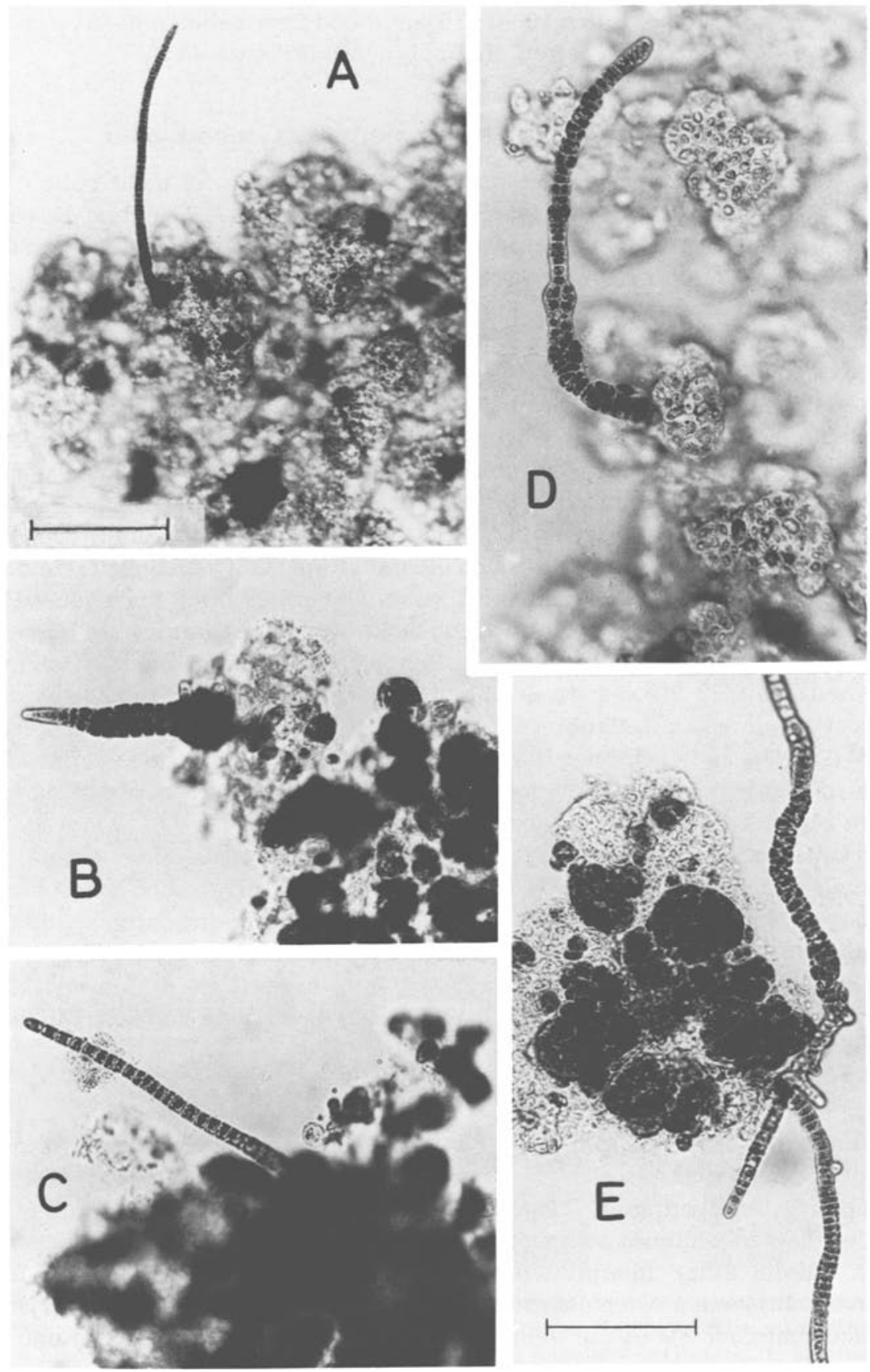

Abb. 6. Erythrotrichopeltis ciliaris. Erythrotrichia-Fäden in einer überständigen Kultur scheibenförmiger Pflanzen. Maßstrecken: $\mathrm{A}=200 \mu \mathrm{m} ; \mathrm{B}-\mathrm{E}=100 \mu \mathrm{m}$ 


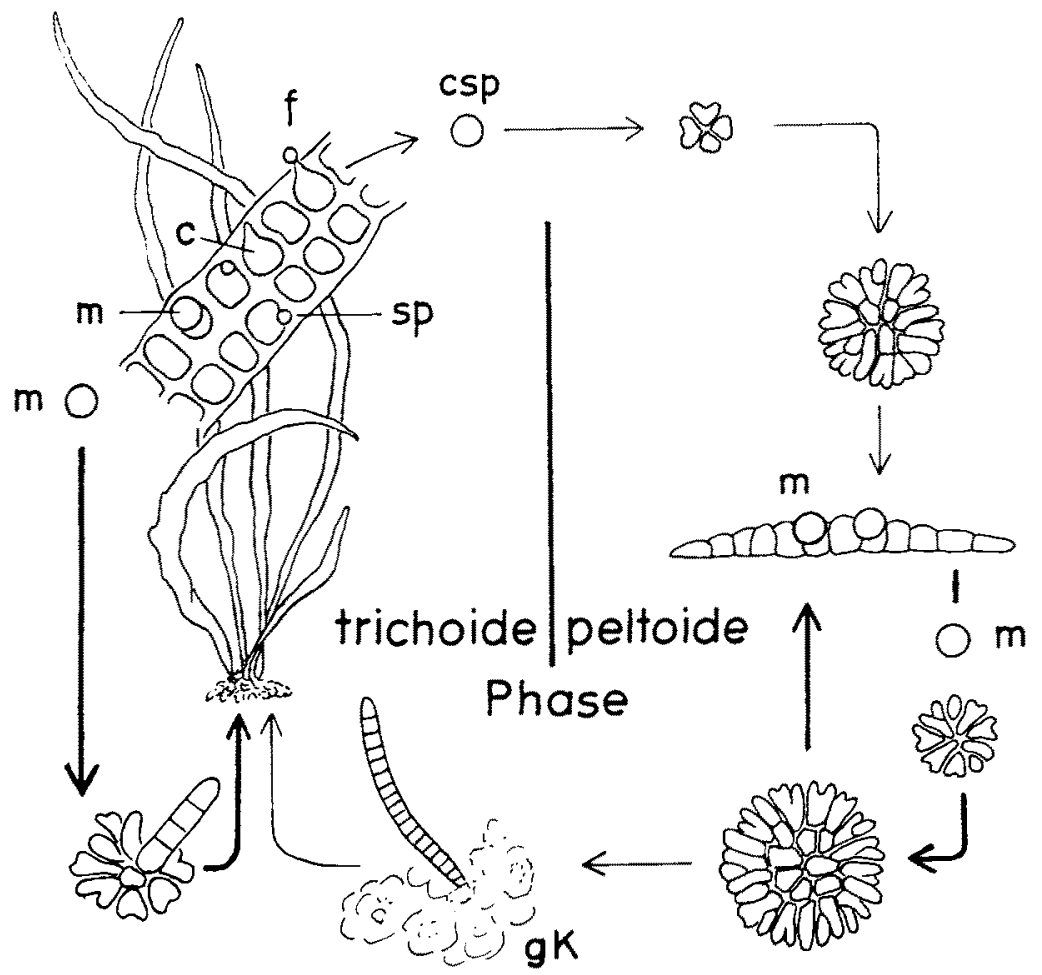

Abb. 7. Die Lebensgeschichte von Erythrotrichopeltis ciliaris schematisch dargestellt. Die trichoide Phase (Erythrotrichia) und die peltoide Phase (Erythropeltis) vermehren sich unabhängig voneinander durch Monosporen (isomorphe Zyklen, dicke Pfeile). Karposporen des monözischen fädigen Thallus leiten den heteromorphen Zyklus ein; gealterte Kulturen der Scheibengeneration führen (nach einer Reduktionsteilung?) zur fädigen Phase zurück (äußerer Kreis, dünne Pfeile). m, Monospore; c, Karpogonium; sp, Spermatium; f, Befruchtung; csp, Karpospore; gK, gealterte Kultur

chia ciliaris (Carm.) schon vor Batters (1900) von Thuret (in Le Jolis, 1863) angewandt wurde, jedoch nicht auf Bangia ciliaris, sondern auf Porphyra boryana begründet. Der zwar durch die Regeln gerechtfertigte, aber dennoch unrichtig angewandte Name Erythrotrichia ciliaris (Carm. ex Harv.) Thuret in Le Jolis trat seitdem bei den Autoren an die Stelle von Erythrotrichia ciliaris (Carm.) Batters.

Die von Heerebout (1968) erstellten Synonymie-Listen sind zu wenig begründet, um hier diskutiert zu werden; Dangeard (1969) hat sich schon kritisch dazu geäußert.

Erythrotrichopeltis ciliaris (Carm. ex Harv. in Hook.) nov. comb.

B a s y o n y m : Bangia ciliaris Carmichael, in Hook. Br. Fl. II, p. 316; Harvey, Phyc. Brit. 1846-51, P1. 322

Sy n on y m e: Erythrotrichia ciliaris (Carm.) Batters, 1900, p. 374

Erythrotrichia discigera Berth., 1882, p. 25, Taf. 1, Fig. 15-18

Erythrotrichia obscura Berth., 1882, p. 26, Taf. I, Fig. 19-25 
Erythropeltis discigera (Berth.) Schmitz, 1896, p. 313, Fig. 195 Erythrotrichia discigera Schmitz var. flustrae Batters, 1900, p. 376 Erythrocladia gibber Dangeard, forme à grandes cellules marginales, 1968 , p. 19, Pl. I, fig. 9-15; Pl. II, fig. 14-23; Pl. VII, A, B; Pl. IX, C

In der Diagnose von Erythrotrichia discigera ist nur die Angabe von Bedeutung: ". .. Fäden . . gruppenweise aus einer einschichtigen Scheibe entspringend, oder letztere allein vorhanden" (Berthold, 1882, S. 25). Nur die eingehende Beschreibung ihrer Fortpflanzung im Text und die Abbildungen vervollständigen die Kennzeichnung dieser Art.

Batters (1900) untersuchte die Originale von Bangia ciliaris Carm. mit dem Ergebnis, daß die Fäden einer gut entwickelten monostromatischen Scheibe entspringen, "but I found many apparently quite mature discs which bore no erect filaments, and which in every way resembled Berthold's figure of the discs of his Erythrotrichia discigera" (p. 375). Der Fundort des Typus ist Appin an der Westküste von Schottland, die Alge wurde auch bei den Scilly Islands und an der schottischen Ostküste gesammelt.

Feldmann (1939) fand Erythrotrichia ciliaris reichlich im Winter und Frühahr bei Banyuls und gibt mehrere Abbildungen. Als unsicher erscheint ihm die Abgrenzung von der ebenfalls dort gesammelten Erythrotrichia obscura. Die nicht konstant bei dieser Art anzutreffende Verzweigung (Børgesen, 1927) kann nicht als unterschiedliches Merkmal angesehen werden.

Dangeard (1932) untersuchte Scheiben von Erythrotrichia discigera auf Bryopsis muscosa. Die bis $200 \mu \mathrm{m}$ im Durchmesser großen fertilen Thalli trugen keine aufrechten Fäden (Pl. XVII, N-R). Sie gleichen, auch in der Größe der Zellen, völlig der von uns kultivierten Scheibengeneration. Durch ihre violette Färbung und die größeren Zellen unterscheiden sie sich gut von der auf demselben Substrat vorkommenden "Erythrocladia" subintegra. Dangeards Zeichnung von Scheiben mit ein- bis dreizelligen aufrechten Fädchen (PI. XVII, Q) ist zu wenig realistisch, um zu überzeugen.

In derselben Arbeit bildet Dangeard (1932, Fig. 1) auch die Basis von Erythrotrichia obscura $\mathrm{ab}$, eine kleine Scheibe, auf der sich aufrechte Fäden erheben. In Kultur erhielt Dangeard (1968) aus epiphytisch auf Bryopsis gewachsenen Thalli ganz diesen entsprechende Pflanzen auf dem Schalenboden (Pl. IV, 1-6).

Die Identität von Erythrocladia gibber, forme à grandes cellules marginales (Dangeard, 1968), mit unserem Erythropeltis-Stadium wurde schon in anderem Zusammenhang (p. 210) erörtert und braucht hier nicht wiederholt zu werden. Dangeard fand dieses Objekt sehr häufig in Kulturen mit Algen aus Guéthary und Biarritz.

Die von Batters (1900) als Erythropeltis discigera Schmitz var. flustrae beschriebenen Scheiben auf Flustra foliacea entsprechen völlig unserem Material. Ihr Fundort Deal nahe Dover - ist das Helgoland nächstgelegene Vorkommen einer der Erscheinungsformen von Erythrotrichopeltis. Die Scheiben trugen keine aufrechten Fäden. Batters' Unsicherheit in der Beurteilung der Alge spiegelt sich in dem Satz wider (S. 376): "I have little or no doubt that this plant is really a form of Schmitz's Erythropeltis discigera; but I cannot think it the same as Erythrotrichia discigera Berthold, although the fronds in many respects resemble the discs of that species when no erect fronds are produced." 
Die von Taylor (1957) für die Nordostküste von Nordamerika angegebene Bangia ciliaris Carmichael weicht von der typischen Art völlig ab. Die Fäden werden $0,5-2 \mathrm{~cm}$ lang, auch ist ihre Basis (Pl. 28, Fig. 5 und 8) nicht scheibenförmig. Ebenso entsprechen die von Kapraun (1980) als Erythrotrichia ciliaris (Carm. ex Harv.) Thur. in Le Jolis in seiner Meeresalgen-Flora von North Carolina aufgeführten, 3-5 cm langen, rosafarben bis braunen, büschelig wachsenden Epiphyten nicht dem europäischen Typus.

\section{Erythrotrichopeltis boryana (Mont.) nov, comb.}

B a sionym: Porphyra boryana Mont., 1849, p. 150, Pl. 13; Ardré, 1970, p. 47, Pl. 4, Fig. 10-11

Sy nonyme: Erythrotrichia boryana (Mont.) Berth., 1882, p. 25

Erythrotrichia ciliaris (Carm.) Thuret in Le Jolis sensu Ardré, 1970, p. 46, Pl. 4, Fig. 6-9

Erythrotrichia tristanensis Baardseth, 1941, p. 33, Fig. A-E

Nach Bertholds Angaben gleicht Erythrotrichia boryana aus dem Golf von Neapel in der Farbe und Größe Erythrotrichia discigera. Der in der Regel einschichtige, oben abgestutzte, nach unten allmählich verschmälerte Thallus ist etwa $5 \mathrm{~mm}$ lang und knapp $0,5 \mathrm{~mm}$ breit.

Ardré (1970) sammelte an der Küste von Portugal eine Alge, die sie mit Vorbehalt zu Erythrotrichia ciliaris (Carm.) stellte, weil die Größe der Zellen nicht die von Batters (1900) und von Feldmann (1939) angegebenen Maße erreicht. Vielmehr entsprechen sie mehr denen des im Herbarium Montagne verwahrten Typusmaterials von Porphyra boryana (Pl. 4, Fig. 10-11). Durch Text und Abbildungen ist die von Ardré gesammelte Alge gut gekennzeichnet; die monosiphon einer geschlossenen Basalscheibe entspringenden Fäden verbreitern sich zu einer monostromatischen Zellfläche mit meist 4 , selten auch 8 bis 9 nebeneinanderliegenden Zellreihen.

Auf Tafel 4, Figs 1-4, gibt Ardré einen als Erythrocladia subintegra Rosenv. bezeichneten scheibenförmigen Thallus wieder. Die fertilen Scheiben sind $30 \mu \mathrm{m}$ dick und haben wesentlich größere Zellen als die typische Art. Zum Vergleich sei auf eine Abbildung bei Dangeard (1932, Pl. XV) verwiesen, in der die Scheibe nur $12 \mu \mathrm{m}$ dick ist. Es liegt nahe, Erythrocladia subintegra sensu Ardré als das scheibenförmige Stadium im Entwicklungszyklus einer Erythrotrichopeltis-Art zu deuten.

Diese Vermutung wird durch einen Befund von Heerebout (1968) gestützt. " . . . in the dried herbarium specimen of Porphyra boryana Montagne [ = E. boryana (Mont.) Berthold] sent by Montagne to Kützing it was possible to find all stages, from a germinating spore growing out into a multicellular disc without filaments up to a multicellular disc with filaments. In Roscoff (France) I was able to collect these stages alive" (p. 145). In einem Kulturversuch mit Sporen von $E$. boryana erhielt Heerebout keine monostromatischen Scheiben, sondern kleine monosiphone Fäden, die aber nach wenigen Tagen abstarben. Auch in unseren Kulturen waren bei großer Sporendichte viele Keimlinge fädig, während aus zerstreut liegenden Sporen selten andere als scheibenförmige Keimlinge entstehen.

Die von Baardseth (1941) beschriebene Erythrotrichia tristanensis wird von Ardré nicht erwähnt. Dabei gleichen die Abbildungen der beiden Autoren einander so sehr, daß an der Identität des ihnen zugrunde liegenden Materials kein Zweifel ist. Baardseth 
bildet rein männliche Fäden sowie solche mit Spermatangien und Monosporangien ab. Karpogonien waren, wie auch nicht anders zu erwarten, nicht zu erkennen. In den häufig gefundenen fertilen Scheiben ohne aufrechte Fäden vermutet Baardseth eher Stadien der Entwicklung von Erythrotrichia tristanensis als selbständige Vertreter der Gattungen Erythrocladia oder Erythropeltis.

Bei einem Vergleich seiner Alge mit den neun bekannten Erythrotrichia-Arten mit Basalscheibe zieht Baardseth drei in die engere Wahl. Die Zellen und Monosporen von E. ciliaris (Carm.) Batters sind etwa doppelt so groß wie bei $E$. tristanensis. (Batters' Angabe von $18 \mu \mathrm{m}$ dicken Sporen darf indessen bezweifelt werden!) Neben der für eine Beurteilung zu unvollkommen beschriebenen $E$. discigera Berth. sieht Baardseth in Erythrotrichia polymorpha Howe eine seiner neuen Art sehr nahestehende Form, von der sie sich jedoch wesentlich in der Größe der Basalscheiben unterscheidet.

\section{Erythrotrichia polymorpha Howe}

Es ist nicht leicht, eine klare Vorstellung von dieser an der peruanischen Küste vorkommenden Art zu gewinnen. Zu sehr "polymorph" erscheinen die Abbildungen auf Howes Tafel 29; Text und Legenden lassen an der Einheitlichkeit des Untersuchungsmaterials zweifeln. Als Typus werden die den Figuren 1-12 sowie 15 und 16 zugrundeliegenden Pflanzen angegeben. Sie wuchsen epiphytisch auf Cladophora und Chaetomorpha, meist als ausgedehnte, $150-600 \mu \mathrm{m}$ große fertile Basalscheiben; nur wenige Scheiben trugen einreihige Fäden von 50-250 $\mu \mathrm{m}$ Länge (Fig. 8 und 11). Ob der in Figur 12 dargestellte vierreihige Faden wirklich zu einer solchen großen Scheibe gehört, kann bezweifelt werden. Er paßt viel besser zu den mehrreihigen Thalli der Figuren 13 und 14 sowie ihrem fertilen Fadenabschnitt in Figur 17. Diese Pflanzen wuchsen auf verschiedenen Braunalgen (Glossophora, Lessonia, Spatoglossum). "The disc, while of the same general character, has a less luxuriant development and the erect filaments attain larger dimensions, becoming finally $850 \mu \mathrm{m}$ long and 2, 4, and occasionally 8 cells in greatest width" (p. 78). Ihre Basalscheiben sind nicht abgebildet, sie waren nicht fertil wie die Scheiben auf Chaetomorpha.

Sehr wahrscheinlich enthält Howes Erythrotrichia polymorpha ein Gemisch zweier Arten. Die in den Figuren 12-14 und 17 abgebildeten Thalli gehören zweifellos zu einer Erythrotrichopeltis-Art. Offensichtlich bezogen sich Baardseth (1941) und Ardré (1970) nur auf diese Thalli bei dem Vergleich mit ihren Aufsammlungen, die untereinander in der Zellengröße gut übereinstimmen. Auch Taylor (1945) identifiziert seine bei den Galapagos-Inseln gesammelten Pflanzen von Erythrotrichia polymorpha mit der von Howe abgebildeten, Erythrotrichia boryana nahestehenden Form. Die Fäden waren 2-2,5 mm lang und verbreiterten sich aus einer einreihigen Basis zu einem 6-8 Zellen breiten Band. "The bases of these plants seem to consist of a few cells loosely arranged, but not in conspicuous disks as figured by Howe" (p. 133). Levring (1960) bearbeitete Expeditionsmaterial von der chilenischen Küste. Sein Befund über Erythrotrichia polymorpha stimmt mit dem von Taylor überein. In der Algenflora der östlichen tropischen und subtropischen amerikanischen Küsten (Taylor, 1960) ist Erythrotrichia polymorpha nicht angegeben.

Der taxonomische Status der von Howe als Typus bezeichneten Pflanze - einreihige, nicht fertile Fäden auf einer auffallend großen Basalscheibe - ist ungeklärt. 


\section{ABSCHLIESSENDE BEMERKUNG}

Es war nicht vorauszusehen, daß sich der zufällige Fund einer unscheinbaren scheibenförmigen Rotalge in einer Rohkultur zu einer solch umfassenden und für unsere Kenntnis der Erythropeltidaceen grundlegenden Studie ausweiten konnte. Wieder einmal hat sich das Kulturexperiment als unverzichtbare Notwendigkeit für die Kennzeichnung kleinerer Algen erwiesen. Mögen die Ergebnisse dieser Arbeit zu entsprechenden Studien mit definiertem, am natürlichen Standort gesammelten Ausgangsmaterial anregen. Eine Mitteilung über andere Vertreter der Erythropeltidaceen von Helgoland wird vorbereitet.

Danksagung. An dem Werden dieser Arbeit nahm Herr Paul-Heinz Sahling mit Freude und Spannung teil. Mit stetem Interesse besorgte er die umfangreiche photographische Dokumentation und fertigte die Abbildungen. Ich danke ihm herzlich für seine Mitarbeit.

\section{ZITIERTE LITERATUR}

Ardré, F., 1970. Contribution à l'étude des algues marines du Portugal. I-La flore. - Port. Acta biol. (B) 10,1-423.

Baardseth, E., 1941. The marine algae of Tristan da Cunha. - Results Norw. scient. Exped. Tristan da Cunha 9, 1-174.

Batters, E. A. L., 1900. New or critical British marine algae. - J. Bot. 38, 369-379.

Berthold, G., 1882. Die Bangiaceen des Golfes von Neapel und der angrenzenden MeeresAbschnitte. - Fauna Flora Golf. Neapel 8, 1-28.

Børgesen, F., 1927. Marine algae from the Canary Islands. III. Rhodophyceae. - Biol. Meddr 6, (6), $1-97$.

Cole, K. \& Conway, E., 1980. Studies in the Bangiaceae: Reproductive Modes. - Botanica mar. 23, 545-553.

Dangeard, P., 1932. Sur quelques Erythrotrichia et Erythrocladia de Banyuls et du Croisic. Botaniste 24, 143-155.

Dangeard, P., 1968. Recherches sur quelques Bangiophycées (Protofloridées). - Botaniste 51, 5-57.

Dangeard, P., 1969. Observations nouvelles sur les Bangiophycées. - Botaniste 52, 5-14.

Dixon, P. S., 1973. Biology of the Rhodophyta. Oliver \& Boyd, Edinburgh, 285 pp.

Dixon, P. S. \& Murray, S. N., 1981. Life histories in the Erythropeltidaceae (Rhodophyta, Bangiophyceae). - Proc. int. Seaweed Symp. 8, 93-97.

Feldmann, J., 1939. Les algues marines de la côte des Albères. IV. Rhodophycées. - Revue algol. 11, 247-330.

Fritsch, F. E., 1945. The structure and reproduction of the algae. Univ. Press, Cambridge, 2, 1-939.

Hamel, G., 1924. Floridées des France - Bangiales. - Revue algol. 1, 278-292; 427-457.

Harvey, H. W., 1851. Phycologia Britannica. London, 3, pl. 241-360.

Heerebout, G, R., 1968. Studies on the Erythropeltidaceae (Rhodophyceae - Bangiophycidae). Blumea 16, 139-157.

Hooker, W. J,, 1833. British Flora. London, 2.

Howe, M. A., 1914. The marine algae of Peru. - Mem. Torrey bot. Club 15, 1-185.

Kapraun, D. F., 1980. An illustrated guide to the benthic marine algae of coastal North Carolina. I. Rhodophyta. UNC Press, Chapel Hill, 206 pp.

Kylin, H., 1956. Die Gattungen der Rhodophyceen. Gleerup, Lund, $673 \mathrm{pp}$.

Le Jolis, A., 1863. Liste des algues marines de Cherbourg. Paris, $168 \mathrm{pp}$.

Levring, T., 1960. Reports of the Lund University Chile expedition 1948-49. 39. Contributions to the marine algal flora of Chile. - Lunds Univ. Ársskr. (N. F., Avd. 2.) 56 (10), 1-85.

Nichols, H. W. \& Lissant, E. K., 1967. Developmental studies of Erythrocladia Rosenvinge in culture. $-J$. Phycol. 3, 6-18. 
Oltmanns, F., 1922. Morphologie und Biologie der Algen. II. Phaeophyceae - Rhodophyceae. Fischer, Jena, 439 pp.

Papenfuss, G. F., 1951. Notes on South African marine algae. III, - Jl S. Afr. Bot. 17, 167-188.

Schmitz, F., 1896. Bangiaceae. In: Die natürlichen Pflanzenfamilien. Hrsg. von A. Engler \& K. Prantl. Engelmann, Leipzig, 1 (2), 307-316.

Taylor, W. R., 1945. Pacific marine algae of the Allan Hancock expeditions to the Galapagos Islands. - Allan Hancock Pacif. Exped. 12, 1-528.

Taylor, W. R., 1957. Marine algae of the northeastern coast of North America. Univ. of Michigan Press, Ann Arbor, 509 pp.

Taylor, W. R., 1960. Marine algae of the eastern tropical and subtropical coasts of the Americas. Univ. of Michigan Press, Ann Arbor, 870 pp. 